\title{
Acquisitions de données topographiques par drone pour la réalisation d'une étude hydraulique complexe
}

\author{
Flavien VIGUIER ${ }^{1}$, Ombeline FOREST ${ }^{2}$
}

${ }^{1 .}$ SNCF Réseau - flavien.viguier@réseau.sncf.fr

2.SNCF Réseau - ombeline.forest@réseau.sncf.fr

\begin{abstract}
RÉSUMÉ. - Afin de contrôler l'intégrité des réseaux hydrauliques, les équipes SNCF réalisent régulièrement des vérifications du dimensionnement des installations. Ces études sont la plupart du temps menées à l'aide de relevés topographiques terrestres (station totale, GPS, ...). Afin de minimiser les coûts d'intervention tout en optimisation la qualité des acquisitions et des rendus, SNCF Réseau a souhaité tester l'utilisation de drones pour l'acquisition des données d'entrée. Le présent article présente la mission réalisée (de l'acquisition de données à la fourniture des livrables de l'étude hydraulique), les travaux de validation des données acquises par drone (en comparant des données photogrammétriques par drone avec des acquisitions LiDAR par ULM) ainsi que l'étude des livrables drones (plan topographique, profils en travers, ...) qui a été menée pour aboutir à la livraison de l'étude hydraulique complète de la zone d'étude.
\end{abstract}

Mots-clés : système ferroviaire, drone, photogrammétrie, LIDAR, étude hydraulique

\section{UAV topographic acquisition for a complex hydraulic study}

\begin{abstract}
To control hydraulic network, SNCF checks regularly sizing of the installations. This kind of study is usually made by using conventional terrestrial topography process (total station, GPS ...). To minimise operation cost while improving acquisitions and deliverables quality, SNCF Réseau has decided to test the use of UAV to acquire input data. This article presents those experimental acquisitions (from data acquisition to hydraulic study deliverables), UAV data validation (by comparing UAV photogrammetric data with ULM LiDAR data) but also UAV deliverables analysis (topographic plan, cross sections...) which was lead to make the hydraulic study of the area of interest.
\end{abstract}

Key-words: Railway System, UAV, Photogrammetry, laser scanning, hydraulic study

\section{INTRODUCTION}

Dans le cadre de la gestion des risques naturels sur les ouvrages en terre (remblais et déblais) ferroviaires, l'approche par la technique d'évaluation des risques type « Nœud Papillon » (cf. Norme ISO 31000 CEI-31010) permet au mainteneur d'identifier les causes et les conséquences des dangers potentiels, et de choisir différentes barrières pour éviter un déraillement ou en diminuer au maximum les conséquences.

Cette approche permet de prendre en compte plusieurs enjeux :

- Le premier porte sur la gestion de l'ouvrage en terre proprement dit (enjeu périmètre métier) ;

- Le second à l'échelle d'un sous-système infrastructure (enjeu périmètre mainteneur) ;

- Le troisième à l'échelle du système ferroviaire global (enjeu périmètre acteurs ferroviaires) qui se rapproche d'un management fondé sur une appréciation qualitative du risque, qui combine évaluation de l'aléa, niveau de vulnérabilité de l'infrastructure et enjeux associés.

Dans le cadre de l'arrivée de la ligne à grande vitesse Bretagne-Pays de Loire (BPL), la politique de l'axe Le Mans-Nantes a fortement été modifiée en termes d'objectifs et de performances. L'analyse de risque sur l'axe a donc été réévaluée, afin de réduire au maximum les impacts au niveau de la sécurité mais aussi de la régularité des circulations ferroviaires.
Une des problématiques de cet axe se situe au niveau des deux Evènements Critiques Redoutés (ECR) suivants : coulées et inondations (ECR2) ainsi que glissements en déblais (ECR3).

Des inondations récurrentes et de nombreux désordres au niveau des déblais sur un tronçon de $3.5 \mathrm{~km}$ ont conduit à la préconisation d'une étude hydraulique complexe afin de mieux gérer l'aléa hydraulique et ainsi réduire les risques tout en minimisant les répercussions sur l'exploitation de la ligne déjà fortement circulée.

Afin de réaliser cette étude hydraulique, un levé topographique précis des emprises ferroviaires et de son environnement était nécessaire. Au regard des contraintes d'intervention (difficulté d'accès aux emprises, étendue de la zone à relever, ...), SNCF a souhaité expérimenter les acquisitions par drone. En effet, les drones, de par leur capacité à mesurer et à inspecter de larges corridors de manière non intrusive (aucun accès aux infrastructures n'est nécessaire pour réaliser les acquisitions) se posent comme une solution de mesure à grande rendement pouvant compléter les méthodes topographiques classiques mais également comme une solution alternative aux levés par méthode aéroportée. En effet, de par leur facilité de mise en œuvre, les aéronefs télépilotés permettent la réalisation, à faible coût et en un temps restreint, de relevés photogrammétriques de larges corridors.

Il est à noter que cette expérimentation par drone s'inscrit dans un programme de recherche interne plus global visant 
l'intégration de ce nouvel outil dans les processus de mesure et de contrôle des infrastructures ferroviaires (surveillance d'ouvrages d'art et d'ouvrages en terre, inspections d'installations hydrauliques, analyse du couvert végétal,...).

Dans le cadre de cette expérimentation, et afin de qualifier le domaine de pertinence des drones tout en assurant la qualité de l'étude hydraulique, les acquisitions par drone (levé réalisé à l'aide d'un capteur photogrammétrique capturant des données dans le visible embarqué sur un drone DT18) ont été comparées à un relevé aéroporté classique combinant acquisitions photogrammétriques et LIDAR. Cet article présente donc l'utilisation opérationnelle des drones dans le cadre d'une étude hydraulique.

\section{OBJECTIF DE L'ETUDE HYDRAULIQUE}

En raison de la modification de la topographie du terrain, suite à la création de la ligne à grande vitesse BPL, des études hydrauliques spécifiques ont été menées afin d'assurer le bon dimensionnement des différents dispositifs de cette ligne nouvelle. Ces études ont été particulièrement poussées au niveau des raccordements ferroviaires visant à interconnecter la ligne nouvelle à la ligne existante car il était nécessaire de contrôler qu'aucun débit supplémentaire n'allait entrer dans le réseau hydraulique existant. Ainsi, l'ensemble des dispositifs hydrauliques de ce raccordement a été dimensionné de façon à n'avoir aucun impact négatif sur la situation hydrologique initiale.

Néanmoins, l'historique d'incidents (glissements, coulées) constatés sur la ligne existante, le nombre d'inondations signalées et la faible capacité hydraulique du réseau de drainage actuellement en place laissent supposer l'apparition de nouveaux désordres. Au vu de la réévaluation de l'analyse de risque sur l'axe Le Mans-Nantes, cette probabilité de désordres n'est pas acceptable; c'est pourquoi SNCF a décidé d'engager des travaux sur ce secteur, nécessitant une étude hydraulique globale.

Cette nouvelle étude a donc pour but d'étudier les options permettant de réduire le risque d'apparition de désordres liés à la présence d'eau dans les déblais et d'inondations sur la ligne classique actuelle. L'étude analysera le fonctionnement et la capacité du réseau existant dans un premier temps, puis, explorera dans un second temps les aménagements capables d'intercepter les eaux de ruissellement en crête des déblais. En effet, compte-tenu de la configuration du site et de la traversée de plusieurs bassins versants naturels par des déblais, il est nécessaire de travailler sur une meilleure gestion de l'aléa, à son origine, en minimisant les répercussions sur l'exploitation de la ligne.

\section{LE CAHIER DES CHARGES}

Le secteur géographique à étudier s'étend sur environ $2.6 \mathrm{~km}^{2}$ soit un corridor de $800 \mathrm{~m}$ de large sur un linéaire de $3.5 \mathrm{~km}$. Ces $2.6 \mathrm{~km}^{2}$ permettent de couvrir l'ensemble des voies ferrées, des différents bassins versants naturels interceptés par la ligne ferroviaire existante et des dispositifs hydrauliques actuellement en place.

Pour la réalisation de l'étude, il est nécessaire d'acquérir des données d'entrée fiables permettant de caractériser avec précision la géographie du site, c'est pourquoi des relevés topographiques tridimensionnels sont nécessaires. De plus, au regard des contraintes d'intervention et des risques hydrauliques potentiels de la zone d'étude, une précision d'acquisition de 5 centimètres en planimétrie et 5 centimètres en altimétrie est requise.

Pour mener à bien leurs études, les hydrauliciens s'appuient principalement sur des données tridimensionnelles (Modèles Numérique de Surface et Modèle Numérique de Terrain), des plans topographiques et des profils en travers. Sur ces différents documents doit être représenté l'ensemble des éléments caractérisant le terrain et pouvant avoir un impact important sur le dimensionnement des ouvrages hydrauliques. En complément, doivent apparaître les installations ferroviaires qui permettent d'assurer la sécurité et la régularité des circulations. En effet, ces installations, qui ne peuvent être modifiées, se posent comme une contrainte lors de la réalisation des travaux (toute modification des installations environnantes peut engendrer des répercussions sur l'exploitation et/ou la maintenance). Connaître la position des infrastructures ferroviaires permet donc d'orienter le choix technique des travaux, en prenant en compte les différentes contraintes de réalisation (analyse à l'échelle du sous-système infrastructure).

Un cahier des charges décrivant les attendus du levé topographique a donc été établi. Ainsi, afin d'assurer la réalisation de l'étude, l'ensemble des éléments présentés ci-dessous est requis (ces éléments doivent pouvoir être représentés sur les différents livrables) :

- le terrain naturel : les talus (crêtes et pied des déblais et remblais) et les lignes de rupture de pente car ces informations sont utiles à la délimitation des bassins versants existants,

- les différents ouvrages et installations hydrauliques : buses, regards, ... afin de caractériser le réseau actuel,

- les parties bâties : les ouvrages d'art, les immeubles et propriétés apparentes des constructions riveraines. En effet, ces données sont indispensables pour identifier les éventuelles contraintes de réalisation (phasage des travaux) mais également pour caractériser les bassins versants,

- les clôture et limites de cultures riveraines complétées par la représentation des éléments cadastraux. Ces données permettent de délimiter le périmètre des emprises SNCF et d'ainsi identifier les zones d'interventions (travaux) possibles,

- les voies de circulations : routes, chemins car la connaissance de la position des accès rend possible l'étude du phasage des travaux (quels engins peut-on faire circuler, comment, ...),

- les installations électriques et de télécommunication. Ces installations fixes, très difficilement modifiables se posent comme une contrainte dimensionnante pour les études et les travaux,

- la végétation : dimension des arbres...

En complément de ces éléments situés dans le domaine public il est indispensable de relever les installations ferroviaires :

- les voies ferrées pour connaître la position exacte des installations actuelles mais également pour localiser le chantier dans les coordonnées ferroviaires (Ligne, Voie et Point kilométrique). Ce système de coordonnées est normé et doit impérativement être utilisé pour tout chantier en milieu ferroviaire, - les installations électriques - poteaux caténaires, regards, armoires et caniveaux électriques, signalisation ferroviaire - car ces installations peuvent représenter des contraintes pour l'étude (réduction de section des ouvrages hydrauliques existants, diminution de l'espace disponible en piste et en crête pour implanter de nouveaux dispositifs, ...),

- les installations hydrauliques : regard, caniveaux. En effet, connaitre l'état actuel du drainage de la plateforme ferroviaire 
est indispensable afin d'évaluer sa capacité hydraulique et caractériser les écoulements au sein de celui-ci.

Sur ce secteur un ensemble varié de cultures, champs, forêts et zones d'habitations est présent, rendant complexe la réalisation d'acquisitions par méthodes terrestres (nombreux masques de mesures, difficultés pour accéder aux propriétés privées situées dans la zone de levé, ...) c'est pourquoi SNCF a souhaité que les travaux de mesures soient réalisés par méthodes aéroportées; la précision de mesure requise (pour rappel 5 centimètres) et l'échelle de restitution souhaitée $\left(1 / 500^{\mathrm{e}}\right)$ étant parfaitement compatibles avec ce type de mesure.

Il est à noter que, conformément au décret n²000-1276 du 26 décembre 2000, le rattachement des levés au système RGF93 en projection Lambert 93 a été exigé.

\section{LES OPERATIONS TOPOGRAPHIQUES DE TERRAIN}

Comme évoqué précédemment, afin de qualifier le domaine de pertinence des drones tout en assurant la qualité de l'étude hydraulique, les acquisitions par drone ont été comparées à un relevé aéroporté classique combinant acquisitions photogrammétriques et mesures LIDAR. Pour garantir la précision et la complétude des levés, ces deux méthodologies d'acquisition ont été combinées à un ensemble d'acquisitions topographiques terrestres.

\section{IV.1. Géoréférencement des levés}

\section{IV.1.1. Contrôle du canevas polygonal existant}

Dans le cadre de la création de la ligne à grande vitesse Bretagne Pays de Loire, un canevas polygonal principal constitué de points géodésiques (connus dans les 3 dimensions $\mathrm{X}, \mathrm{Y}, \mathrm{Z}$ ) répartis tous les 500 mètres linéaires a été établi. Pour faciliter les opérations de géoréférencement tout en assurant la cohérence planimétrique et altimétrique des levés aéroportés avec l'existant, SNCF a fourni au prestataire réalisant les acquisitions, la société Redbird, les coordonnées des différents points de ce canevas.

Néanmoins, afin de valider la précision des coordonnées fournies, et de respecter les spécifications techniques du cahier des charges (précision des levés de $5 \mathrm{~cm}$ en planimétrie et 5 centimètres en altimétrie) SNCF a imposé un contrôle de ces données par l'intermédiaire d'acquisitions GPS post-traitées rattachées au Réseau GNSS Permanent (RGP) de l'Institut Géographique National (IGN). Les écarts observés entre coordonnées initiales et coordonnées de contrôle étant tous inférieurs à 2 centimètres le canevas polygonal principal a été validé.

\section{IV.1.2. Mise en place de points de contrôle au sol}

Comme évoqué précédemment, au regard des contraintes d'acquisition en milieu ferroviaire (impossibilité d'accéder aux emprises) les données topographiques seront acquises par photogrammétrie aérienne (par drone et système aéroporté). Pour assurer la qualité des ortho photographies qui seront produites à partir des différents clichés acquis, la mise en place de points de contrôle au sol, appelés GCP (Groud Control Point) est nécessaire. Ces GCP ont été matérialisés au sol sur des surfaces stables et dégagées afin qu'ils soient visibles par le drone et qu'ils soient utilisables lors du traitement de données ; les formes, couleurs et dimensions des GCP ont également été soigneusement sélectionnées (cf. figure 1).

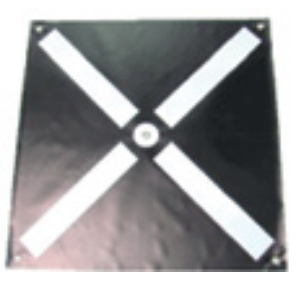

Figure 1 : Matérialisation d'un GCP (@Redbird).

Ces points qui permettent d'éliminer les déformations liées à la création des livrables photogrammétriques sont également utilisés pour valider le géoréférencement des données. Au regard de l'étendue de la zone de levé, de la topographie du site et des exigences de précision, la mise en place de points tous les 500 mètres linéaires a été décidée. De plus afin d'éviter toute distorsion dans les livrables (orthophoto, MNT, ...) ces points ont été répartis sur l'ensemble de la zone de levé (points en limite mais également au cœur de la zone d'étude). Il est à noter qu'en raison de l'impossibilité d'accéder aux emprises ferroviaires, les points de contrôle ont été implantés sur le domaine public. La figure 2 présentée ci-dessous illustre la répartition des GCP.

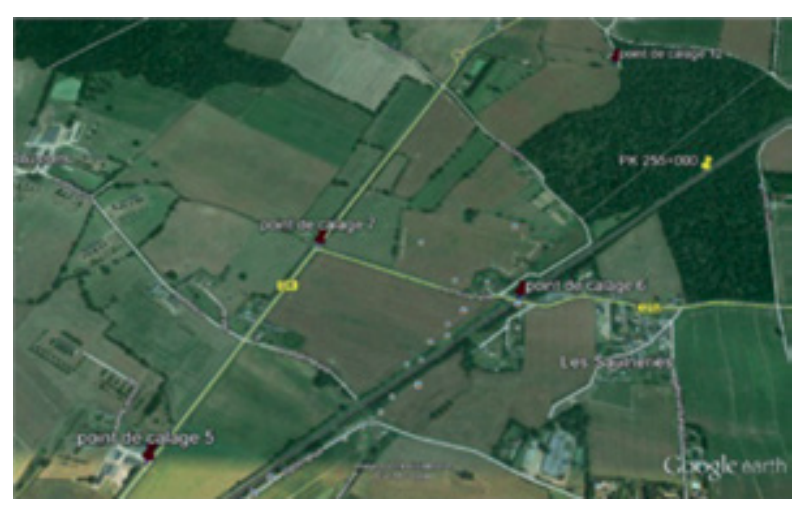

Figure 2 : Répartition des points de contrôle (@Redbird).

Une fois positionnés ces points ont été déterminés par mesures GPS temps réel. Pour cela, un capteur GNSS (Global Navigation Satellite System) Trimble GeoExplorer SPS 985L a été utilisé et a permis d'obtenir les coordonnées des GCP avec une précision meilleure que 5 centimètres. Pour valider la précision de mesures, un contrôle sur les points du canevas polygonal principal, précédemment validé, a été effectué.

\section{IV.1.3. Réalisation des acquisitions aériennes}

Une fois les GCP implantés, les acquisitions topographiques aériennes ont pu être réalisées. Pour cela, la société a mobilisé deux plateformes volantes distinctes :

- un drone à voilure fixe Delair Tech DT 18 (cf. figure 3) équipé d'une caméra photogrammétrique de 5 Mégapixels. Ce vecteur a été sélectionné afin de couvrir en un seul vol l'ensemble de la zone de levé (pour assurer la cohérence des acquisitions mais également pour limiter les contraintes et les risques d'intervention au niveau d'infrastructures ferroviaires exploitées). En effet, ce drone pesant moins de 


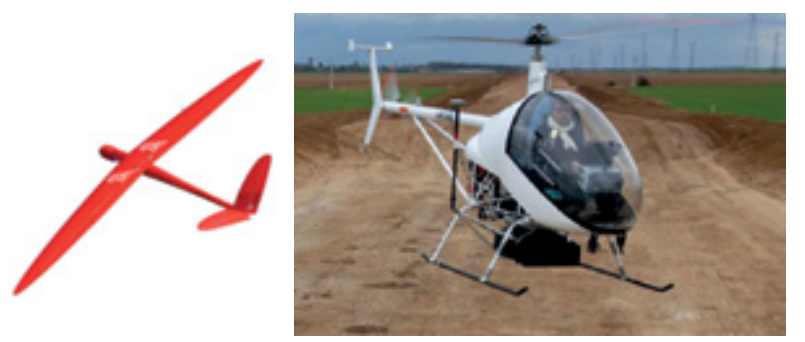

Figure 3 : Le drone Delair Tech DT18 opéré par la société Redbird (à gauche) et l'ULM équipé d'un capteur photogrammétrique et d'un capteur LIDAR (à droite) (@Redbird).

2 kilogrammes est validé par la DGAC en scénario S4 ce qui permet la réalisation d'acquisitions hors vue du télépilote.

- un Ultra Léger Motorisé (ULM), de marque Kompress, équipé d'un capteur photogrammétrique de 80 Mégapixels couplé à un LIDAR RIEGL VQ480U de précision $2.5 \mathrm{~cm}$ et capable d'acquérir jusqu'à 275000 points par seconde (cf figure 4). Cette configuration a été sélectionnée en raison de son faible coût d'intervention mais également de la qualité des capteurs embarqués. En effet, le RIEGL VQ 480-U est un système LIDAR a retour d'onde complète qui permet la réalisation de mesures y compris sous couvert végétal. Une grande partie de la zone d'intervention étant recouverte de végétation (végétation basse, arbres, ...) le choix de cette configuration de mesure était indispensable afin d'acquérir précisément le Modèle Numérique de Terrain.

Pour chacune des méthodologies de mesures, des demandes d'autorisation de vol ont été déposées auprès des autorités compétentes (DGAC, DSAC). Ces demandes étaient constituées d'un dossier présentant une analyse des cartes aériennes mais également les plans de vol projetés (cf figure 4).

Il est à noter qu'afin d'assurer la précision de mesure ainsi qu'un recouvrement important entre les images acquises (recouvrement de $70 \%$ en longitudinal et $30 \%$ en transversal) tout en respectant la règlementation aérienne, la hauteur de vol maximale sur la zone d'étude a été fixée à 150 mètres par rapport au sol. Un exemple d'image acquise à l'aide du drone ainsi qu'un extrait de nuage de points mesuré par l'ULM sont présentés en figure 5 .

Bien que très performantes les méthodologies de mesure aéroportées sont soumises à un ensemble de contraintes météorologiques fortes. En effet, en présence de vent fort

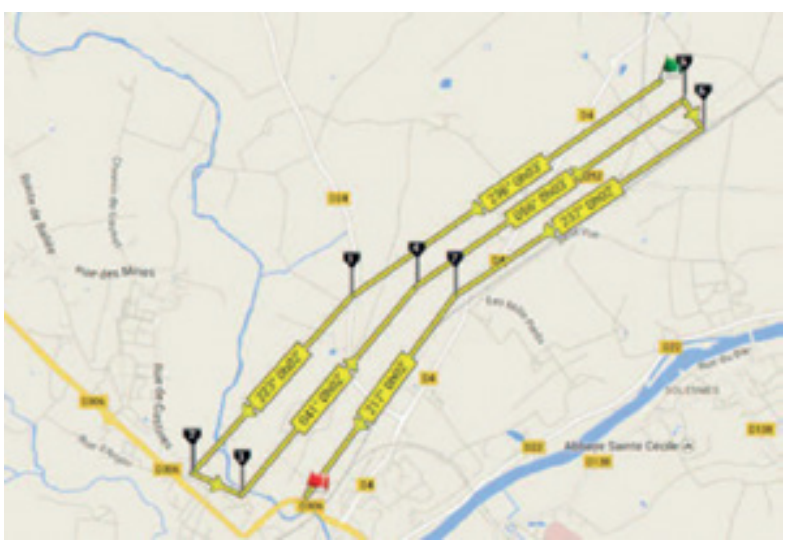

Figure 4 : Carte aéronautique Mach 7 itinéraire du drone (@)Redbird).

ou de pluie, il est impossible de mettre en œuvre les acquisitions ce qui peut être problématique lors de la réalisation de mesures en urgence (après un incident ou un évènement météo). De plus, afin d'obtenir des données de qualité, les acquisitions doivent être effectuées en prenant en considération la position du soleil :

- si les photos sont réalisées en présence d'un soleil bas sur l'horizon des ombres seront constatées sur les clichés (ombres pouvant masquer des éléments importants pour l'étude),

- si les conditions d'éclairement varient au cours des mesures, des différences radiométriques seront observées sur les clichés ; variations radiométriques pouvant interdire la production des ortho photographies et des nuages de points photogrammétriques.

Ainsi, les opérations de mesures ne sont déclenchées qu'en présence de conditions météorologiques optimales (peu de vent, éclairement constant) et lorsque le soleil est à son zénith (absence d'effet d'ombre sur les images).

\section{IV.1.4. Réalisation de compléments terrestres}

Afin de connaitre avec exactitude l'état du réseau hydraulique existant des mesures topographiques terrestres complémentaires ont dû être réalisées. En effet, bien que quasi exhaustives les mesures aéroportées (LIDAR ou photogrammétriques) ne fournissent pas de données suffisamment précises sur la position des fils d'eau ou des regards hydrauliques. De plus, les méthodologies aériennes ne permettent pas d'obtenir des informations sur les ouvrages situés sous
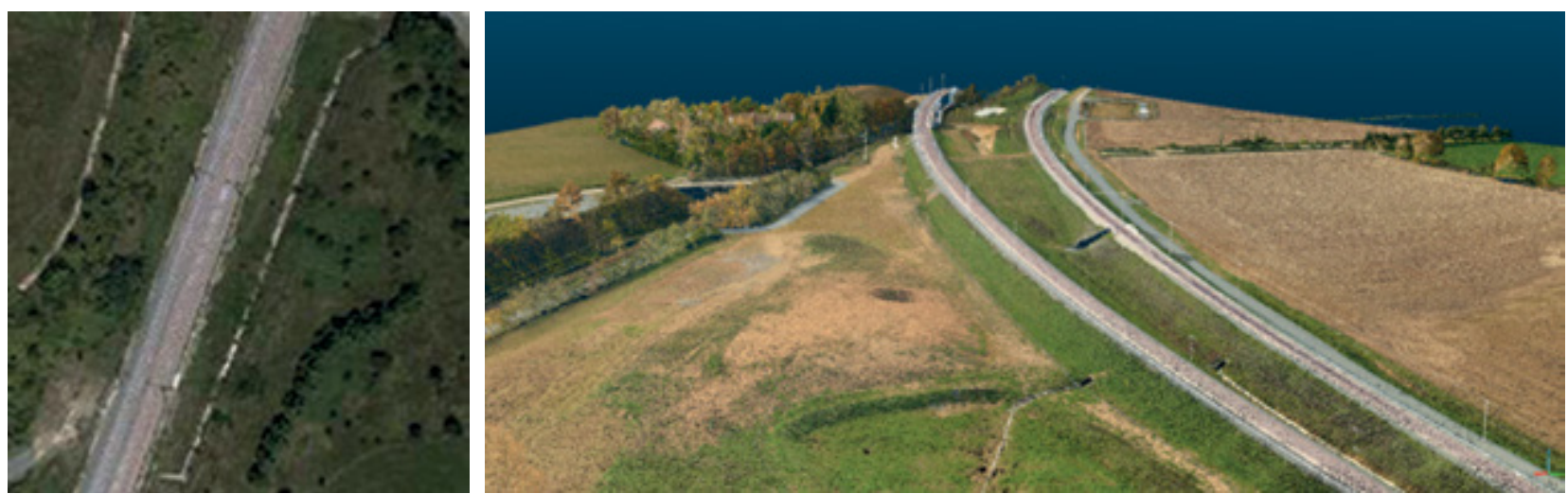

Figure 5 : Exemple d'image acquise par le drone sur la partie gauche et nuage de points LIDAR sur la partie droite (@Redbird). 
voies (buses, aqueducs, ...). En se basant sur le canevas polygonal principal de la ligne à grande vitesse BPL, les géomètres de la société Redbird ont donc réalisé des mesures par tachéométrie pour compléter les données aériennes. Au-delà de leur intérêt pour la détermination des réseaux hydrauliques, ces mesures topographiques ont également permis le levé des lignes de ruptures de pentes au niveau des déblais et des remblais, informations qui seront utilisées lors de la création du Modèle Numérique de Terrain.

\section{LES TRAITEMENTS TOPOGRAPHIQUES}

Afin de restituer les documents requis par le cahier des charges, Redbird a réalisé un ensemble de traitements sur les trois sources de données : la photogrammétrie par drone, le nuage de points LIDAR couplé à la photogrammétrie acquis par ULM et le levé topographique par tachéométrie classique. Les analyses et traitements effectués ont permis la fourniture du Modèle Numérique de Terrain, du plan topographique et des profils en travers de la zone d'étude.

Les différentes données acquises ont bien évidemment étaient géo référencées ; pour cela les informations de positionnement fournies par la centrale inertielle et le GPS (de l'ULM ou du drone) ont été utilisées. En complément, les GCP ont été exploités pour effectuer un « recalage » des données.

\section{V.1. Levé topographique terrestre}

Pour permettre la réalisation du MNT, Redbird a tout d'abord extrait les données topographiques acquises par levé terrestre. En effet, comme évoqué précédemment, ces levés ont permis de relever les lignes de rupture de pente ainsi que l'ensemble des réseaux hydrauliques présents sur site (fossés, buses, ...) ; informations indispensables au calcul de dimensionnement des installations en place et futures.

Deux techniques pouvaient être envisagées : utilisation $\mathrm{du}$ nuage de points LIDAR ou création d'un nuage de points par corrélation dense d'images (en se basant sur les photographies acquises par drone ou par l'ULM). Au regard de la végétation présente sur le site, l'utilisation des données LIDAR a été retenue car le RIEGL VQ 480-U, LIDAR a retour d'onde complète, permet l'acquisition de données y compris sous couvert végétal. En effet, en filtrant les différents échos laser reçus par le capteur il est possible d'extraire automatiquement la végétation et donc de déterminer le sol nu (cf. figure 6).
Les informations sur le sol nu ont alors été complétées par les lignes de rupture de pente acquises par topographie classique (apport de contraintes de restitution) pour obtenir le MNT final représentant de manière quasi exhaustive et avec une précision meilleure que 5 centimètres l'ensemble des formes et dimensions de la zone d'étude.

Il est à noter que le nuage de points LIDAR a également été colorisé à partir des clichés acquis simultanément lors de la campagne de mesure ULM (cf figure 5). Cette colorisation du nuage permet une visualisation plus aisée des données et présente une aide utile lors du report des différents objets mesurés.

\section{V.2. Fourniture des orthophotos}

Après avoir corrigé les clichés photogrammétriques acquis à l'aide du drone (intégration des distorsions radiales et tangentielles, ...), Redbird a pu démarrer la production des ortho photographies. Afin de limiter toute déformation dans les orthophotos, une correction complémentaire des images a été appliquée en exploitant le semis de points du sol nu calculé précédemment à l'aide du LIDAR.

Une fois les orthophotos produites et corrigées géométriquement différentes corrections radiométriques ont été appliquées : réduction des anomalies de contraste dans les images, égalisation radiométriques sur l'ensemble des images utilisées et rehaussement global des couleurs. Est alors obtenu l'ortho photographie finale pouvant être directement exploitée pour réaliser le plan de la zone d'étude.

Il est à noter que des traitements identiques ont été réalisés à partir des clichés photographiques acquis à l'aide de l'ULM. En effet, c'est à partir des orthophotos produites à l'aide des deux systèmes de mesure que sera réalisée l'une étude comparative (analyse des distorsions dans les images, comparaison de la taille des pixels objets, ...).

\section{V.3. Réalisation des plans}

A partir des ortho photographies et du MNT, la restitution des plans topographiques a pu être réalisée : l'utilisation des clichés permet de comprendre les éléments à restituer alors que le MNT est utilisé pour la création des polylignes 3D. Les données issues du relevé topographique classique ont bien évidement été utilisées pour restituer précisément les réseaux hydrauliques. La combinaison de ces trois sources de données permet donc le respect des précisions et des attendus du cahier des charges.

Une fois le plan topographique produit, des profils en travers de la zone, tous les 20 mètres, ont été réalisés. C'est
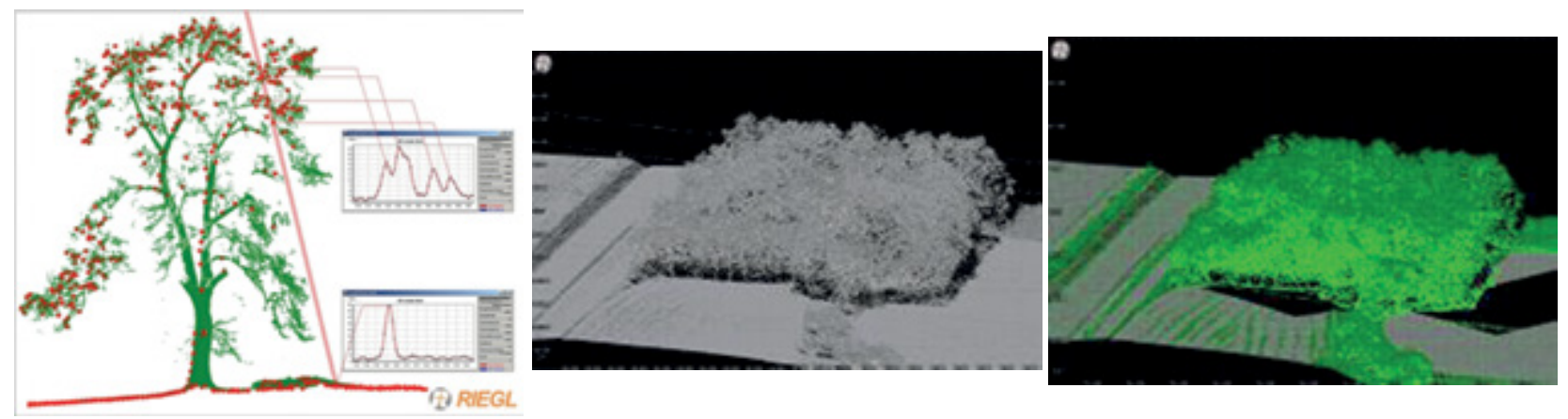

Figure 6 : Principe de fonctionnement d'un LIDAR à retour d'onde complète (@Riegl) à gauche, nuage de points LIDAR brut au centre et filtrage de la végétation à droite. 
à partir de ces profils, combinés au MNT et au plan topographique que sera réalisée l'étude hydraulique.

\section{ETUDE HYDRAULIQUE}

Les acquisitions topographiques serviront de données d'entrée pour les étapes 1 à 3 de l'étude hydraulique, présentées dans la suite de ce document. Elle sera appréhendée de manière classique.

\section{VI.1. Analyse fonctionnelle hydraulique}

Une description synthétique du fonctionnement hydraulique des ouvrages de la ligne existante (en intégrant l'infrastructure du raccordement BPL) sera réalisée ainsi qu'un recensement des installations présentes dans l'environnement immédiat (superstructure ferroviaire, réseaux traversant, voirie) dans le but de réaliser une analyse fonctionnelle globale (interception des bassins versants, interaction réseau hydraulique $\mathrm{SNCF} / \mathrm{tiers}$ ).

\section{VI.2. Etape hydrologique}

L'étude hydrologique permettra d'identifier les bassins versants interceptés par les déblais puis d'estimer des débits générés par ces surfaces pour des périodes de retours courantes et exceptionnelles.

\section{VI.3. Construction d'un modèle hydraulique}

Le modèle hydraulique permettra d'évaluer la capacité du réseau existant puis de tester des scénarii d'aménagement pour améliorer les caractéristiques du système. Il sera adopté une combinaison d'approches en utilisant des calculs et/ou modèles simples ou des logiciels plus complexes en fonction des solutions proposées. Une modélisation hydrodynamique sera vraisemblablement nécessaire.

\section{VI.4. Analyse de risque hydraulique}

Une analyse de risque préliminaire pour l'état initial sera réalisée afin d'identifier les risques principaux en termes notamment de Fiabilité, Disponibilité, Maintenabilité et Sécurité pour le tronçon de ligne. Les résultats de la modélisation hydraulique pour l'état initial seront utilisés pour évaluer les risques de désordres pour les ouvrages en terre (érosion du talus, glissement, coulée, ...), pour la plateforme (fréquence de débordement du système de drainage et circulation d'eau dans de la plateforme) et la voie (en termes de pollution voire emportement de la couche de ballast). L'analyse de risque permettra d'identifier les zones les plus vulnérables dans le système actuel et d'orienter le choix des aménagements pour les traiter.

\section{VI.5. Solutions d'aménagements}

Plusieurs solutions d'aménagements hydrauliques seront proposées en fonction des résultats des étapes précédentes et la configuration du site. Chaque solution sera étudiée dans le modèle hydraulique pour évaluer son efficacité dans l'amélioration des conditions hydrauliques dans les déblais. Le coût des options sera estimé en termes de «Life Cycle Cost » (coût sur la durée de vie de l'ouvrage) avec une estimation pour les travaux initiaux puis la maintenance sur une période d'évaluation de 50 ans.

\section{CONCLUSION}

L'utilisation de drones dans le cadre d'études hydrauliques se pose comme une solution alternative permettant une acquisition de données à moindre coût. Ce mode d'acquisition est particulièrement intéressant lors de la réalisation d'interventions en milieu complexe où les mesures terrestres sont difficiles à réaliser voire impossible (ex : difficulté d'intervention en voie, et plus généralement dans les emprises ferroviaires, sur une ligne fortement circulée et à vitesse élevée : coupure de ligne, intervention de nuit, multiplication du personnel pour assurer la sécurité, ...).

A la date d'écriture du présent article, nous n'avons pas encore pu comparer la précision des données acquises par l'ULM avec celle du drone mais nous pouvons d'ores et déjà affirmer que les acquisitions photogrammétriques par drone permettent la réalisation de livrables avec une précision de l'ordre de $5 \mathrm{~cm}$. Pour atteindre cette précision, il est néanmoins nécessaire de mettre en place un processus strict de mesure combinant points de contrôles au sol et mesures topographiques terrestres complémentaires.

Il est toutefois à noter qu'en présence d'une forte végétation (forêt, buissons recouvrant le terrain naturel) la seule réalisation d'acquisitions photogrammétriques par drone n'est pas adaptée. En effet, dans le cadre d'une étude hydraulique, une connaissance parfaite du terrain naturel est nécessaire pour modéliser l'environnement et les simples acquisitions photographiques ne sont pas suffisantes (les photographies permettent uniquement de modéliser le couvert végétal et aucune information sur le sol nu n'est fourni). Dans cette situation il est préférable de mettre en œuvre des acquisitions LIDAR. A ce jour aucun système drone équipé d'un LIDAR capable de réaliser des vols hors vue du télépilote n'est autorisé à voler sur le territoire national ; les futures évolutions de la législation drone permettront peut-être de pallier à ce manque. Ce marché étant prometteur, les constructeurs de LIDAR tels que RIEGL, VELODYNE, ... se penchent d'ores et déjà sur la création de LIDAR légers pouvant être installés sur ce type d'aéronef.

Bien que l'utilisation de drones présente de nombreux avantages (coût, rapidité d'intervention, capacité à réaliser des acquisitions de manière non intrusive, ...) cette méthode peut parfois être difficile à déployer et nécessite une préparation stricte : une étude des cartes aériennes doit être réalisée en amont afin de savoir s'il est possible de mettre en œuvre des acquisitions drones (cette étude qui doit être menée par le donneur d'ordres peut être réalisée à l'aide des logiciels Mach ou AIP Drones), une analyse météorologique doit être effectuée (privilégier les acquisitions hors période à forte pluviométrique et à vent fort) et il est indispensable d'anticiper les acquisitions (la durée pour l'obtention des autorisations peut varier entre quelques jours et plusieurs semaines).

\section{REFERENCES ET CITATIONS}

AIP DRONES@ @ 2014] ; Logiciel AIP Drones ; http://www. aip-drones.fr/

Décret $n^{\circ} 2000-1276$ du 26 décembre 2000 portant application de l'article 89 de la loi $\mathrm{n}^{\circ} 95-115$ du 4 février 1995 modifiée d'orientation pour l'aménagement et le développement du territoire relatif aux conditions d'exécution et de publication des levés de plans entrepris par les services publics

MACH $7 @$ [2014] ; Logiciel Mach 7 ; http://www.mach7.com/

Norme ISO 31000 CEI-31010

Principe de fonctionnement d'un LIDAR à retour d'onde complète [Riegl, 2014]. 